Obere Extremität 2013 $\cdot 8: 190$

DOI 10.1007/s11678-013-0228-y

Online publiziert: 10. September 2013

(c) Springer-Verlag Berlin Heidelberg 2013

\section{Martin Richter}

Malteser Handzentrum, Malteserkrankenhaus Bonn-Rhein-Sieg, Bonn, Deutschland

\title{
M. Dupuytren
}

Über viele Jahre schien die Behandlung des M. Dupuytren geklärt zu sein und interessierte die handchirurgische Fachwelt wenig. Die partielle Aponeurektomie und Strangresektion war die allgemein akzeptierte Methode der Wahl. Die familiäre Häufung in bestimmten ethnischen Gruppen belegte die im wesentlichen erbliche Determination.

Die Zulassung der Kollagenase als nichtoperative Methode zunächst im Februar 2010 in den USA und im Mai 2011 in Deutschland hat das Interesse der Handchirurgen geweckt und die Diskussion erneut entfacht. Die Operation war auf einmal nicht mehr die einzig denkbare Therapie. Dass die Kollagenase wirkt, konnten Studien zeigen. Bezüglich der Indikation ist die Diskussion jedoch noch in vollem Gange. Sozusagen im Windschatten der Bewerbung der Kollagenase als nichtoperative Behandlung kam es auch zu einer Wiederbelebung der Nadelfasziotomie als nichtoperative Behandlungsmethode des M. Dupuytren.

Das derzeitige Dilemma der Handchirurgie ist, dass im Gegensatz zu den gut dokumentierten Ergebnissen der Kollagenasebehandlung keine wirklich validen Daten für die Aponeurektomie und Strangresektion sowie die Nadelfasziotomie vorliegen. Dieser Umstand ist vermutlich der Tatsache geschuldet, dass die Zulassung eines Medikamentes wie der Kollagenase eine vorgeschriebene Studienlage erfordert, wohingegen ein solches Verfahren für Operationsmethoden nicht vorgeschrieben ist. Der Vergleich der Behandlungsergebnisse verschiedener Patientengruppen in einschlägigen Fachzeitschriften ist wenig aufschlussreich, da die Patienten oft hinsichtlich Stadium und Diathesegrad schlecht beschrieben sind. Jedem ist klar, dass ein 3bis 4-gradiger Dupuytren mit 3-Fingerbefall bei einem 24-Jährigen mit familiärer Belastung nicht mit einem 2-gradigen umschriebenen Zentralstrang am Ringfinger eines 70-Jährigen verglichen werden kann. Dies wurde in früheren Publikationen nicht ausreichend beachtet.

McGrouther wies schon darauf hin, dass eine erfolgreiche Dupuytrenbehandlung nicht nur die Entfernung des Dupuytrengewebes, sondern auch die Behandlung eines etwaigen Hautdefizites und möglicher Gelenkkontrakturen beinhaltet. Bei minimalinvasiv behandelten Patienten - unabhängig davon, ob sie mit Kollagenase oder Nadelfasziotomie behandelt werden - sind die letztgenannten Probleme nur sehr begrenzt zu lösen. Dem gegenüberzustellen ist die Frage, ob alle Patienten auf völlig gerade Finger angewiesen sind oder ob nicht bei bestimmten Patienten eine partielle Verbesserung des Streckdefizits schon zu einer nahezu vollständigen Funktionswiederkehr führt.

Die Frage des Rezidivrisikos der verschiedenen Verfahren ist auch noch nicht abschließend geklärt. Ein wesentliches Problem ist dabei die genaue - oder besser gesagt - eine allgemein gültige oder akzeptierte Definition des Rezidivs. Solange in Veröffentlichungen die Definitionen zwischen „Auftreten eines Knotens an derselben Hand" und „erforderliche Revisionsoperation an gleicher Stelle innerhalb von 2 Jahren" liegen, ist keine Vergleichbarkeit gegeben. Da bei den minimalinvasiven Verfahren prozedurimmanent Dupuytrengewebe zurückgelassen wird, ist der Rezidivbegriff nur funktionell sinnvoll zu definieren. Die Wiederkehr eines Streckdefizits in einem bestimmten Ausmaß (Gradzahl) bezogen auf ein Fingergelenk in einem definierten Zeitraum wäre klarer. Erste Anstrengungen in diese Richtung wurden schon bei einem Konsensustreffen unternommen. Da zwar vermutlich alle Methoden das Metakarpophalangeal (MP)-Gelenk besser korrigieren als das proximale Interphalangeal (PIP)Gelenk, ist die unterschiedliche Evaluie- rung der Ergebnisse von MP- und PIPGelenken wünschenswert. Offen bleibt jedoch die Frage, in welchem Ausmaß sich dort die Verfahren unterscheiden.

Auch auf der Seite der Ätiologie wurden neuerere Erkenntnisse publiziert. Die molekulare Medizin und Genforschung haben es möglich gemacht, 6 Genloci zu differenzieren, in denen sich die Dupuytrenpatienten von der nichtbetroffenen Bevölkerung unterscheiden. Auf dem Chromosom 7 findet sich bei Dupuytrenpatienten gehäuft eine Sequenz, die auf den Wnt-Signalweg einwirkt. Der Weg von dort zum Dupuytrengewebe ist jedoch noch unklar. Die unterschiedlichen Genloci mit einer Häufung auf Chromosom 7 könnten auch genetischen und damit vielleicht auch klinischen Subtypen entsprechen.

Das scheinbar abgearbeitete Thema der Dupuytrenkontraktur wirft wieder viele spannende offene Fragen auf, die nicht nur der Grundlagenforschung, sondern vor allem im therapeutischen Bereich einer Vielzahl klinischer vergleichender Studien bedürfen. Dieses Themenheft kann diese Fragen nicht beantworten, soll Ihnen aber aktuelle Informationen näher bringen und Einstieg in eine interessante Diskussion und eventuell eigene klinische Forschung sein.

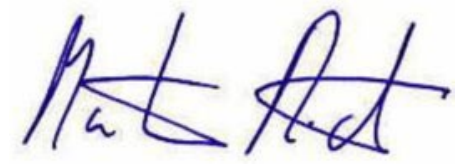

Martin Richter

\section{Korrespondenzadresse}

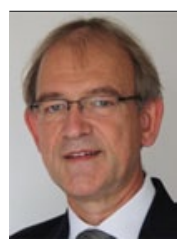

Dr. med. M. Richter

Malteser Handzentrum

Malteserkrankenhaus Bonn-

Rhein-Sieg

Von-Hompesch-Str. 1

53123 Bonn

martin.richter@malteser.org 